\title{
Cerebral radiation necrosis complicating stereotactic radiosurgery for arteriovenous malformation
}

\author{
P Statham, P Macpherson, R Johnston, D M Forster, J Hume Adams, N V Todd
}

\begin{abstract}
A patient presented with symptoms and signs of raised intracranial pressure and increasing focal deficit 13 months after stereotactic radiosurgical treatment of an arteriovenous malformation (AVM). Computed Tomography (CT) showed a mass lesion at the site of the previous abnormality typical of radiation necrosis, but with features identical to those of a malignant neoplasm. Biopsy confirmed cerebral radiation necrosis. The radiation dose was 25 Gray to the periphery of two overlapping $14 \mathrm{~mm}$ collimator fields, delivered in a single dose. Treatment with steroids led to improvement in the symptoms and signs of raised intracranial pressure, but not the focal deficit. Radiation necrosis is a consequence of the large doses required to obliterate AVMs and is a limiting factor in their treatment. It is important for clinicians referring patients for stereotactic radiosurgery to be aware of this complication, and to be able to recognise and treat it.
\end{abstract}

Late radiation necrosis that occurs after cranial irradiation was first described in 1930 by Fischer, ${ }^{1}$ and has been extensively reported since..$^{2-17}$ There is only one report of radiation
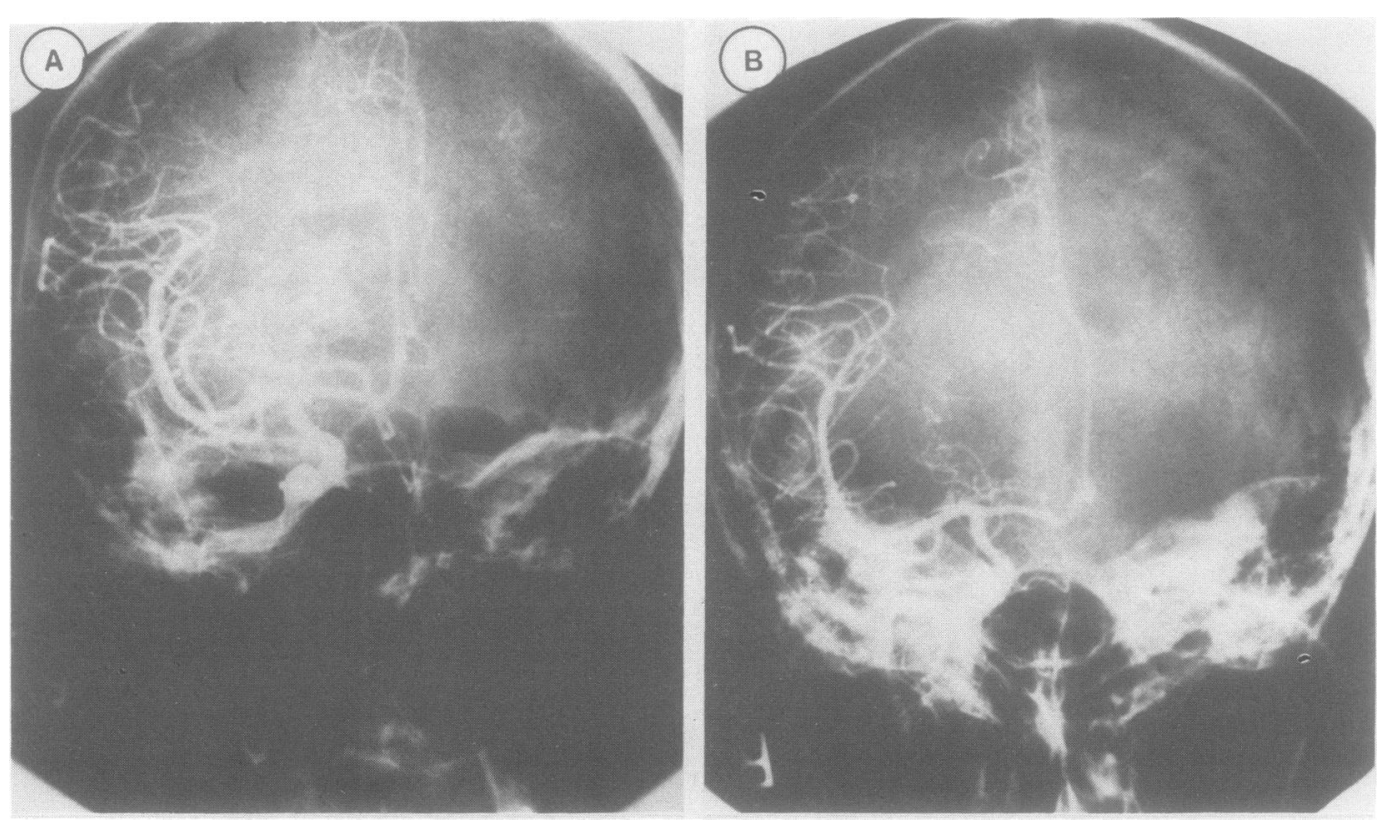

Figure 1 Right Carotid Angiogram, (a) AVM fed by anterior and middle cerebral arteries. (b) 13 months after treatment, demonstrating almost complete obliteration of the AVM, and an avascular mass between the anterior and middle cerebral vessels.

necrosis after stereotactic radiosurgery for AVMs, affecting three of 135 patients treated in Sweden, ${ }^{18}$ and this is the first report of radiation necrosis following AVM radiosurgery in the United Kingdom, documented with both radiology and histology.

\section{Case report}

An eighteen year old girl presented in 1965 with sudden severe headache and drowsiness, while she was 36 weeks pregnant. She had a dense left hemiparesis and a temporal visual field deficit. Cerebral angiography showed an AVM in the region of the right basal ganglia, supplied by both anterior and middle cerebral arteries, with a small contribution from the posterior cerebral artery. She was not offered operation, and improved, but was left with a mild hemiparesis. Twenty two years later she developed sudden headache and worsening of the left hemiparesis. Computed tomography (CT) showed calcification in the region of the right basal ganglia, and haematoma extending into the lateral ventricle. Angiography showed a $14 \mathrm{~mm}$ by $22 \mathrm{~mm}$ by $30 \mathrm{~mm}$ AVM (fig la). Operation was declined, and she was referred for stereotactic radiosurgery which was performed at the national centre in Sheffield. Two overlapping $14 \mathrm{~mm}$ collimator fields were used to deliver 25 Gray to the periphery of the lesion in a single dose.

Received 10 May 1989 and in revised form 20 October

Accepted 10 November 1989 
Figure 2 CT. (a) 3 months after treatment calcification in the right basal ganglia region and surrounding region of low attenuation. (b) 13 months after treatment; peripherally contrast enhancing mass, with surrounding low attenuation region and mass effect. (c) 18 month after treatment; resolution in mass effect and

reduction in low

attenuation region size.
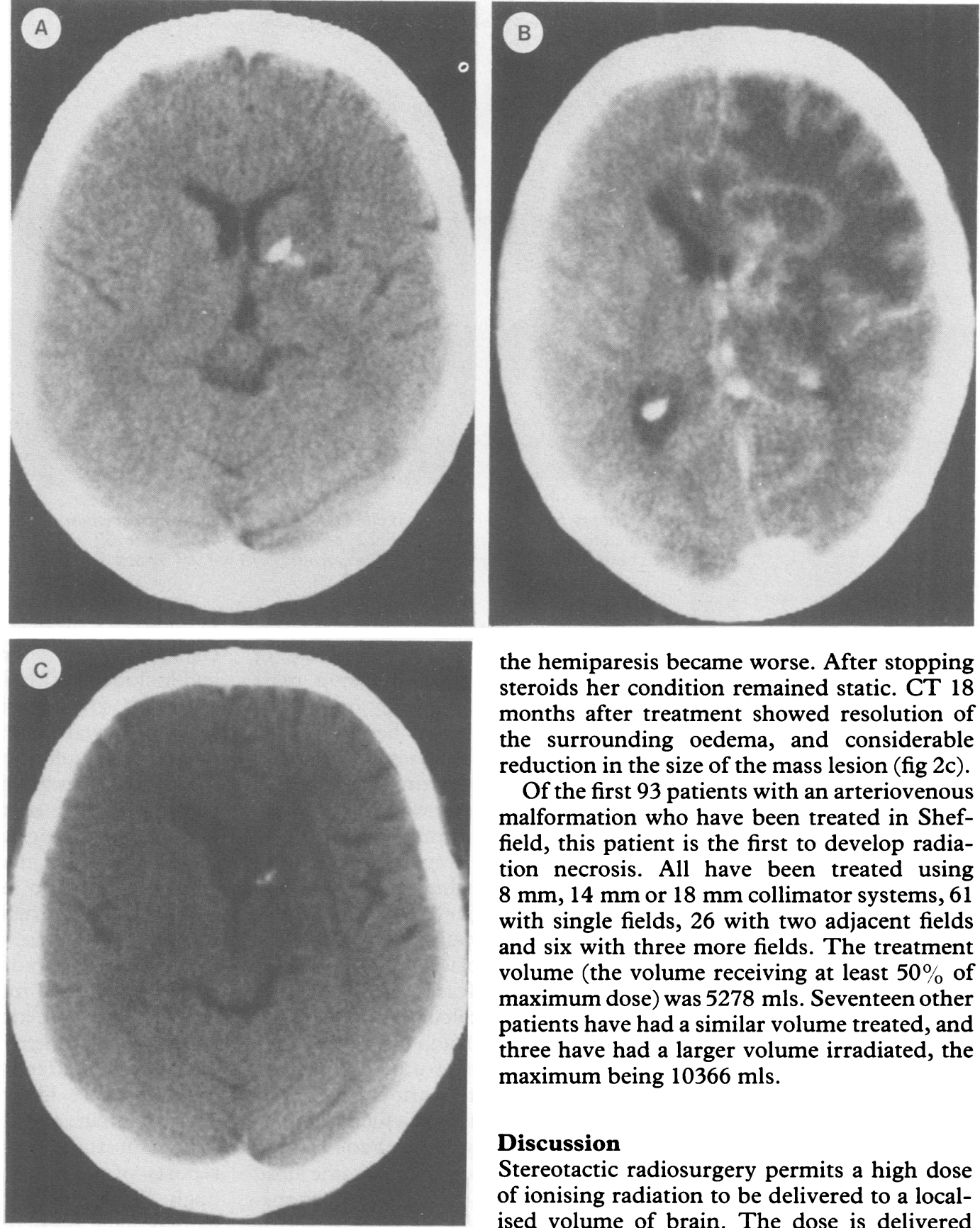

the hemiparesis became worse. After stopping steroids her condition remained static. CT 18 months after treatment showed resolution of the surrounding oedema, and considerable reduction in the size of the mass lesion (fig $2 \mathrm{c}$ ).

Of the first 93 patients with an arteriovenous malformation who have been treated in Sheffield, this patient is the first to develop radiation necrosis. All have been treated using $8 \mathrm{~mm}, 14 \mathrm{~mm}$ or $18 \mathrm{~mm}$ collimator systems, 61 with single fields, 26 with two adjacent fields and six with three more fields. The treatment volume (the volume receiving at least $50 \%$ of maximum dose) was $5278 \mathrm{mls}$. Seventeen other patients have had a similar volume treated, and three have had a larger volume irradiated, the maximum being $10366 \mathrm{mls}$.

\section{Discussion}

Stereotactic radiosurgery permits a high dose of ionising radiation to be delivered to a localised volume of brain. The dose is delivered using precisely collimated narrow beams of
Thirteen months after receiving radiosurgery she developed headache, vomiting, reduced short term memory, increased left hemiparesis and bilateral papilloedema. At the site of the previous AVM the CT showed an irregular peripherally contrast enhancing mass with extensive white matter oedema and midline shift (fig 2b). Angiography showed complete obliteration of the AVM (fig 1b), except for a small residuum fed by the posterior cerebral artery. As the CT appearances were compatible with either radiation necrosis or a malignant tumour, stereotactic biopsy was performed. Histology showed the typical features of cerebral radiation necrosis; endothelial proliferation, intramural thrombus, perivascular lymphocyte cuffing and fibrinoid necrosis (fig 3 ).

She was treated with dexamethasone for three months. The symptoms and signs of raised intracranial pressure disappeared, but radiation, enabling a steep dose gradient to be achieved. Sharply circumscribed lesions can be produced, with little exposure of adjacent tissue. Stereotactic radiosurgery has been used to treat intracranial tumours such as pituitary adenomas, pinealomas, acoustic schwannomas and meningiomas, as well as functional disorders such as intractable pain, trigeminal neuralgia, Parkinson's disease and epilepsy. More recently it has been used to treat surgically inaccessable AVMs; in a series of 104 patients with AVMs, Leksell described total obliteration in $87 \%$ of patients two years after treatment. $^{18}$

Three groups of brain reactions to external beam irradiation are described: acute reactions which occur during treatment; early delayed reactions which occur a few weeks to three months after completion of radiotherapy, and late delayed reactions which occur from several months to many years later. ${ }^{16}$ Acute and early 


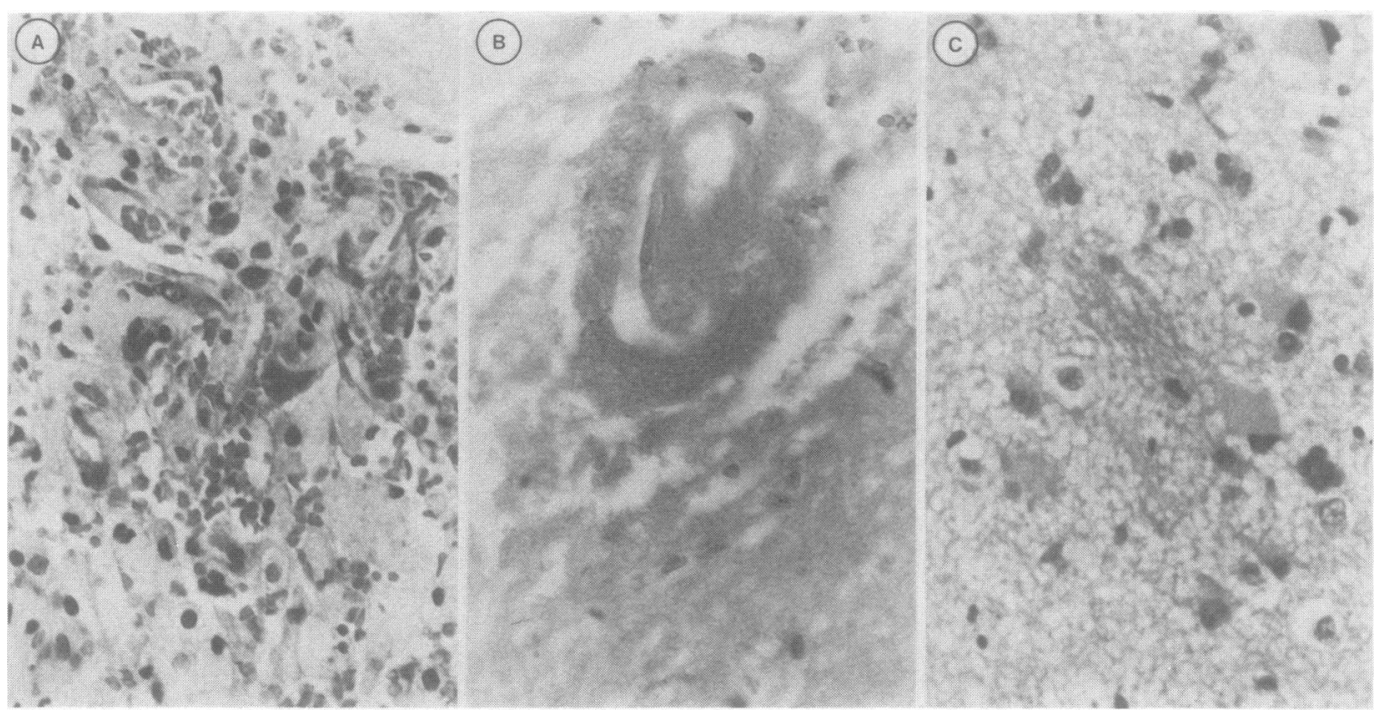

Figure 3 Biopsy Specimen (Haematoxylin and Eosin) from the contrast enhancing rim of the lesion showing; (a) $(\times 530)$ enhanced vascularity, endothelial hyperplasia and perivascular cells with hyperchromatic nuclei. (b) $(\times 530)$ thickened vessel wall with surrounding tissue necrosis, $(c)(\times 750)$ intense astrocytosis.

delayed reactions are usually self limiting. Late delayed reactions are of two types; loss of volume due to cerebral atrophy, or a mass lesion, clinically and radiologically mimicking a malignant tumour. Both may be progressive or irreversible. The three cases reported by Steiner, following stereotactic radiosurgery, presented with mass lesions which responded to steroids, or were self limiting. ${ }^{19}$

There are many case reports of cerebral radiation necrosis following external beam radiotherapy, but few correlate the number of cases affected with the population treated. Risk factors for radiation necrosis include total dose, treatment time and dose fractions. Using these three parameters Ellis calculated a Nominal Standard Dose (NSD) to standardise the biological effect of different treatment regimes. This has been modified by various authors to estimate the threshold above which cerebral radiation necrosis occurs. ${ }^{20-23}$ Patient risk increases with higher dose, or the same dose given in a shorter period, or in fewer fractions. Stereotactic radiosurgery uses large doses of irradiation (up to $100 \mathrm{Gray}$ ) to small volumes of tissue (up to $30 \mathrm{~mm}$ diameter) in a single fraction. The dose per unit volume of tissue, even at the periphery of the lesion, is many times greater than that used in conventional external beam irradiation. This is likely to lead to more cases of brain necrosis, albeit confined to a small volume of tissue. The three previously reported cases received doses of more than 60 Gray to a single field, or more than 40 Gray to two overlapping fields. ${ }^{19}$

The response of blood vessels to irradiation consists of reactive proliferation of the endothelial lining and subendothelial connective tissue, which may subsequently obliterate the lumen. This is the basis of the therapeutic effect of radiation on AVMs. ${ }^{19}$ Similar vascular features are seen in delayed cerebral radiation necrosis; thickened vessel walls, endothelial proliferation, fibrinoid necrosis, hyaline and amyloid deposition, perivascular lymphocytic infiltration and intraluminal thrombosis in medium and small sized arteries. Parenchymal changes occur predominantly in white matter, and include coagulative necrosis, cystic cavitation with gliosis and patchy demyelination. The pathogenic mechanism is probably primarily vascular, with necrosis and glial loss secondary to ischaemia, ${ }^{21124}$ but three other hypotheses have been proposed; direct injury to glial cells, particularly oligodendroglia, may produce white matter degeneration and demyelination, ${ }^{6}$ an autoimmune reaction may result from endothelial damage (vasculitis) or glial antigen release following glial injury, or intracellular free radicles induced by radiation may damage lipid membranes, producing cell membrane dysfunction and cell death. ${ }^{25}$

The development of a mass lesion at least three months after radiation treatment for an AVM is more likely to be due to radiation necrosis than a radiation induced intrinsic malignant tumour. There are only 17 case reports of post-irradiation gliomas, ${ }^{26}$ although fibrosarcomas and malignant meningiomas have been reported more commonly. Biopsy may be required to make the diagnosis if malignant glioma, radiation necrosis or abscess cannot reliably be distinguished by CT or magnetic resonance imaging. Steroids are the only useful medical treatment, and may partly protect against late necrosis if given at the time of irradiation. ${ }^{22}$ Resection of accessible regions of focal necrosis can be worthwhile, ${ }^{17}$ but is unlikely to be of use in patients with partly obliterated, surgically inaccessible AVMs.

With the increasing use of localised high dose radiotherapy, such as stereotactic radiosurgery, proton beam therapy, and interstitial brachytherapy, radiation necrosis will be seen more often. Careful follow up of all patients having treatment is important to determine the true incidence of radionecrosis; modification of treatment doses and volumes may be required. In the United Kingdom, patients with AVMs treated with radiosurgery are referred to a few specialised centres for treatment, but are followed up in peripheral units. It is important for 
those clinicians involved in the long term follow up of these patients to be familiar with the presentation and management of radiation necrosis, as the prognosis is relatively good if only small volumes of brain have been irradiated.

We thank Professor David Graham for his help and advice in preparing the histology.

1 Fischer AW, Holfender H. Lokale Amyloid in Gehirn. Eines Spatfolge von Rontgenbestrahlungen. Deutsch $Z$ Chir 1930;227:475-83.

2 Pennybacker J, Russell DS. Necrosis of the brain due to radiotherapy. Clinical and pathological observations. Neurol Neurosurg Psych 1948;11:183-98.

3 Dugger GS, Stratford JG, Bouchard J. Necrosis of the brain following Roentgen irradiation. Am J Roentgen 1954; 72:953-60.

4 Lindgren M. On tolerance of brain tissue and sensitivity of brain tumours to irradiation. Acta Radiol (Supp) brain tumours

5 Crompton D, Layton DD. Delayed radionecrosis of the brain following therapeutic $\mathrm{X}$ irradiation of the pituitary. Brain 1961;84:85-101.

6 Lampert PW, Davis RL. Delayed effects of radiation on the human central nervous system. Neurology 1964;14:912-7.

7 Kramer S. The hazards of therapeutic irradiation of the human central nervous system. Clin Neurosurg 1968;15:301-18.

8 Kusske JA, Williams JP, Garcia JH, Pribam HN. Radiation necrosis of the brain following radiotherapy of extracerebral neoplasms. Surg Neurol 1976;6:15-20.

9 Takeuchi J, Hanakita J, Abe M, Handa H. Brain necrosis after repeated radiotherapy. Surg Neurol 1976;5:89-93.

10 Martins AN, Johnston JS, Henry JM, Stoffel TJ, DiChiro G. Delayed radiation necrosis of the brain. J Neurosurg 1977;47:336-4

11 Rottenburg DA, Chernik NK, Deck MDF. Cerebral necrosis following radiotherapy for extracerebral neoplasms. Ann Neurol 1977;1:339-57.

12 Michael MA. Radiation necrosis of the brain. Correlation between computed tomography, pathology and dose dis- tribution. J Comput Asst Tomogr 1978;2:71-80.

13 Dilorenzo N, Nolletti A, Palma L. Late cerebral radionecrosis. Surg Neurol 1978;10:281-90.

14 Burger PC, Mahaley MS, Dvoka L, Vogel FS. The morphological effects of radiation administered therapeutically for intracranial gliomas. A post mortem study of 25 cases. Cancer 1979;44:1256-72.

15 Yamashita J, Handa H, Yumitori K, Abe M. Reversible delayed radiation effect on the brain after radiotherapy of malignant astrocytoma. Surg Neurol 1980;13:413-17.

16 Sheline GE. Irradiation injury of the human brain. A review of clinical experience. In: Kagan AR, Gilbert HA, eds. Radiation necrosis of the brain. New York, Raven Press 1980:39-57.

17 Glass JP, Te-Long Hwang Leavens ME, Linshitz HI Cerebral Radiation necrosis following treatment of extracranial malignancies. Cancer 1984;54:1966-72.

18 Leksell D. Stereotactic Radiosurgery. Neurol Res 1987;9: $60-8$.

19 Steiner L. Treatment of arteriovenous malformations by radiosurgery. In: Wilson CB, Stein BM, eds. Intracranial arteriovenous malformations. Baltimore and London: Williams and Wilkins, 1984:295-313.

20 Soffietti R, Sciolla R, Giordana MT, Vasario E, Schiffer D. Delayed adverse effect after irradiation of gliomas: clinicopathological analysis. J Neurooncology 1985;3: 187-92.

21 Sheline GE, Wara WM, Smith V. Therapeutic irradiation and brain injury. Int $J$ Radiat Oncology Biol Phys 1980;6:1215-28.

22 Marks JE, Baglan RJ, Prassad SC, Blank WF. Cerebral Radionecrosis: incidence and risk in relation to dose, time, fractionation and volume. $J$ Radiation Oncology Biol Phys $1981 ; 7: 243-52$.

23 Pezner RD, Archambeau JO. Brain tolerance unit, a method to estimate risk of radiation brain injury for various dose schedules. Int J Radiat Oncol Biol Phys 1982;7:397-402.

24 Hopewell JW, Wright EA. The nature of latent cerebral irradiation damage and its modification by hypertension.

25 Raleigh JA, Kremers W, Gaboury B. Dose rate and oxygen effects in models of lipid membranes: linoleic acid. Int $J$ Radiat Biol 1977;31:203-13.

26 Walker JS, Bigner D. Radiation induced brain tumours. In: Wilkins RH, Rengachary SS, eds. McGraw Hill, Neurosurgery, vol 1. McGraw Hill, 1985:525-8.

27 Judge MR, Eden OB, O'Neill P. Cerebral glioma after prophylaxis for acute lymphoblastic leukaemia. $\mathrm{Br} M e d J$ 1984;289:1038-9. 